\title{
Biomasa del fitoplancton eucariota y su disponibilidad para la red trófica del embalse Riogrande II (Antioquia, Colombia)
}

\author{
Mónica Tatiana López-Muñoz ${ }^{1, *}$, John Jairo Ramírez-Restrepo ${ }^{1}$, Jaime Alberto Palacio-Baena², \\ Ricardo O. Echenique ${ }^{3}$, Carlos E. De Mattos-Bicudo ${ }^{4}$, Edison Andrés Parra-García ${ }^{1}$ \\ ${ }^{1}$ Grupo de Limnología Básica y Experimental y Biología y Taxonomía Marina, \\ Instituto de Biología, Universidad de Antioquia, Medellín, Colombia \\ ${ }^{2}$ Grupo de Investigación en Gestión y Modelación Ambiental, \\ Facultad de Ingeniería, Universidad de Antioquia, Medellín, Colombia \\ ${ }^{3}$ Facultad de Ciencias Naturales y Museo, (UNLP)/CIC-BA, La Plata, Argentina \\ ${ }^{4}$ Núcleo de Pesquisa em Ecologia, Instituto de Botânica, São Paulo, Brasil
}

\begin{abstract}
Resumen
Con el objetivo de establecer la biomasa de carbono contenida en el fitoplancton eucariota del embalse Riogrande II (Antioquia, Colombia), entre agosto de 2011 y agosto de 2012, la porción de esa biomasa disponible para el zooplancton filtrador y la calidad de alimento que podría representar para dicho grupo, el fitoplancton se clasificó en dos rangos de longitud correspondientes a nanoplancton (5 a $20 \mu \mathrm{m}$ ) y microplancton ( $>20 \mu \mathrm{m}$ ). Para ambos grupos se calculó el contenido de carbono celular y la biomasa total en contenido de carbono. Además, para el nanoplancton, el cual corresponde a la fracción filtrable, se estimaron las concentraciones de nitrato y de fosfato. Los altos niveles de biomasa de organismos fototróficos evidenciaron el papel fundamental del fitoplancton eucariótico en la fijación de carbono. Sin embargo, debido a la eutrofización del sistema y a que la talla del zooplancton dominante es pequeña, el flujo de carbono y de nutrientes parece estar dirigido principalmente hacia la vía detrítica y las sustancias disueltas, y no directamente al zooplancton filtrador, cuya principal fuente de alimento en el fitoplancton eucariótico la constituyen los organismos nanoplanctónicos mixotróficos (Cryptophyta y Euglenophyta), que en función de un mayor volumen poseen concentraciones más elevadas de nitrato y fosfato y, por lo tanto, pueden proveer alimento de mejor calidad que el resto del nanoplancton. (C) Acad. Colomb. Cienc. Ex. Fis. Nat. 2016.
\end{abstract}

Palabras clave: biomasa, carbono, fitoplancton, microplancton, nanoplancton, zooplancton.

Eukariotic phytoplankton biomass and its availability for the food web at Riogrande II reservoir (Antioquia, Colombia)

\begin{abstract}
In order to establish the carbon biomass content in the eukaryotic phytoplankton at Riogrande II reservoir (Antioquia, Colombia) from August 2011 to August 2012, as well as the portion of this available biomass for filter-feeding zooplankton and the quality of food it could represent for each group, we classified phytoplankton considering two length ranges corresponding to nanoplankton (5 to $20 \mu \mathrm{m})$ and microplankton $(>20 \mu \mathrm{m})$. We estimated the cellular carbon content and the total biomass represented as carbon content for both groups. We also estimated nitrate and phosphate concentrations for the nanoplankton, which corresponds to the filterable fraction. The high levels of phototrophic organisms biomass showed the essential role of eukaryotic phytoplankton in carbon fixation; however, due to the eutrophication of this reservoir and to the small size of dominant zooplankton, the carbon flux and nutrients seemed to be directed mainly to the detrital track and to dissolved substances and not directly to the filter-feeding zooplankton, whose main food source among eukaryotic phytoplankton is constituted by mixotrophic nanoplanktonic organisms (such as Cryptophyta and Euglenophyta), that according to their greater volume have higher concentrations of nitrate and phosphate and, therefore, can provide better quality food than the remaining nanoplankton. (C) Acad. Colomb. Cienc. Ex. Fis. Nat. 2016.
\end{abstract}

Key words: Biomass, carbon, phytoplankton, microplankton, nanoplankton, zooplankton.

\section{Introducción}

Como fijadoras de carbono y productoras de biomasa, las algas, así como las macrófitas y las bacterias fotosintéticas, son uno de los principales grupos de organismos autótrofos en los ambientes acuáticos continentales (Bellinger \& Sigee,
2010). De esta manera, las algas del fitoplancton tienen un papel fundamental en las redes tróficas acuáticas como productores primarios, ya que son el principal proveedor de energía y nutrientes para los consumidores de niveles tróficos superiores (Moss, et al., 2003). 
La cuantificación de la biomasa de microorganismos presentes en los ecosistemas acuáticos es fundamental en la evaluación de su capacidad de carga, de los requerimientos de nicho y de los flujos de materia y energía trófica. La biomasa es un indicador del éxito de cada especie para reproducirse y sobrevivir a factores de estrés fisiológico y, en general, es una medida de la conversión exitosa de carbono inorgánico en carbono orgánico (Vollenweider, 1969).

Dado que la densidad, o el carbono orgánico total en partículas, no permiten una estimación adecuada de la concentración de partículas disponibles como alimento para los herbívoros (Rocha \& Duncan, 1985), la estimación de la biomasa mediante el carbono se utiliza ampliamente para cuantificar el metabolismo de la comunidad y la transferencia de energía trófica, por lo cual se ha convertido en una herramienta esencial en los estudios funcionales de los ecosistemas acuáticos (Gosselain \& Hamilton, 2000).

Además, la biomasa estimada como el contenido de carbono de los organismos planctónicos es una variable significativa en los modelos de ecosistemas y del equilibrio del carbono biogeoquímico, debido a que este elemento es el componente estructural más importante de los organismos. Generalmente, esta biomasa se estima mediante la conversión del tamaño microscópico al volumen celular (biovolumen) y, posteriormente, a la concentración de carbono, utilizando la derivada de carbono a relaciones de volumen (MendenDeuer \& Lessard, 2000). De esta forma, algunas especies de poco biovolumen pueden cumplir una función muy relevante en el mantenimiento de las estructuras comunitarias a través de los flujos de carbono (Rodríguez-Martínez, 2010).

En este contexto se planteó la pregunta que originó el presente trabajo: ¿qué porción de la biomasa de carbono contenida en el fitoplancton eucariótico del embalse Riogrande II puede ser consumida por el zooplancton dominante en este sistema? Se planteó que si más del $90 \%$ de la biomasa del fitoplancton eucariótico (estimada como biovolumen) fue aportada por especies del género Staurastrum (la morfología de este taxón le permite disminuir el pastoreo), la porción disponible debía ser muy baja (inferior al $5 \%$ de la biomasa estimada como contenido de carbono), y estaría conformada principalmente por especies de Chlorophyta y Cryptophyta, que son las algas dominantes que más fácilmente consume el zooplancton.

Para corroborar la hipótesis, el objetivo de este trabajo fue establecer la biomasa (estimada como el contenido de carbono) en el fitoplancton eucariótico del embalse Riogrande II, así como la porción de dicha biomasa disponible para el zooplancton filtrador y la calidad del alimento que podría representar para este grupo.

\footnotetext{
*Correspondencia:

Mónica Tatiana López-Muñoz, monicatatiana@gmail.com

Recibido: 11 de febrero de 2016

Aceptado: 31 de marzo de 2016
}

\section{Materiales y métodos}

El embalse Riogrande II está ubicado a 2.150 msnm al noroccidente de la ciudad de Medellín (Antioquia, Colombia), en jurisdicción de los municipios de Don Matías, San Pedro, Belmira, Santa Rosa de Osos y Entrerríos, y se sitúa en las coordenadas $75^{\circ} 32^{\prime}-75^{\circ} 26^{\prime}$ O y $6^{\circ} 33^{\prime}-6^{\circ} 28^{\prime} \mathrm{N}$. El embalse tiene un volumen de 240 millones de $\mathrm{m}^{3}$ hasta la cota de vertedero $(2.270 \mathrm{msnm})$, una profundidad máxima de $50 \mathrm{~m}$, un tiempo de retención aproximado de 72,8 días y un caudal aproximado de $35 \mathrm{~m}^{3} \mathrm{seg}^{-1}$ en la captación. Capta las aguas de un área media de drenaje de 1.214 hectáreas, y recibe principalmente las aguas de los ríos Grande y Chico y de la quebrada Las Ánimas. El embalse tiene múltiples usos: generación hidroeléctrica, abastecimiento de agua potable y contribución al saneamiento del río Medellín; además, en su área de influencia se desarrollan actividades agrícolas y ganaderas.

La información se recolectó en cinco estaciones de muestreo localizadas al ingreso (1) y al final (2) del brazo del río Chico, en la torre de captación (3), al final del brazo de la quebrada Las Ánimas (4) y al final del brazo del río Grande (5) (Figura 1).

\section{Muestreo y análisis de las muestras}

Entre agosto de 2011 y agosto de 2012 se hicieron muestreos mensuales en el embalse. Las muestras de fitoplancton se recolectaron en tres profundidades de la zona fótica utilizando una botella van Dorn de $5 \mathrm{l}$ de capacidad, y se fijaron con solución de Lugol y ácido acético al $1 \%$.

La identificación taxonómica del fitoplancton se hizo en un microscopio fotónico usando, entre otras, las claves de Bourrelly (1970), Castro \& Bicudo (2007), Comas (1996), Cox (1996), Gasse (1986), Huber-Pestalozzi (1968), Komárek \& Fott (1983), Prescott, et al. (1972), Prescott, et al. (1975), Prescott, et al. (1982), Teiling (1967), Tell \& Conforti (1986) y West, et al. (1923). El material se depositó en la colección del Laboratorio de Limnología Básica y Experimental y Biología y Taxonomía Marina de la Universidad de Antioquia, Colombia (CLUA).

Para determinar las características morfométricas de las especies se emplearon fotomicrografías y el programa de morfometría geométrica TPSdig2, y los organismos se consideraron como la unidad fisiológica (células, cenobios, colonias y filamentos). Se estimaron el número de células por colonia y las dimensiones de cada organismo, incluida la máxima dimensión lineal (MDL, $\mu \mathrm{m})$. Además, se calcularon el biovolumen $\left(\mathrm{V}, \mu \mathrm{m}^{3}\right)$ y la superficie $\left(\mathrm{S}, \mu \mathrm{m}^{2}\right)$ de acuerdo a las ecuaciones geométricas de Hillebrand, $\boldsymbol{e t}$ al. (1999). El conteo del fitoplancton se hizo mediante la técnica de campos al azar usando cámaras de sedimentación de $50 \mathrm{ml}$ (Lund, et al., 1958; Utermöhl, 1958). Los valores obtenidos en los conteos se convirtieron a densidad utilizando la fórmula de Ros (1979). 


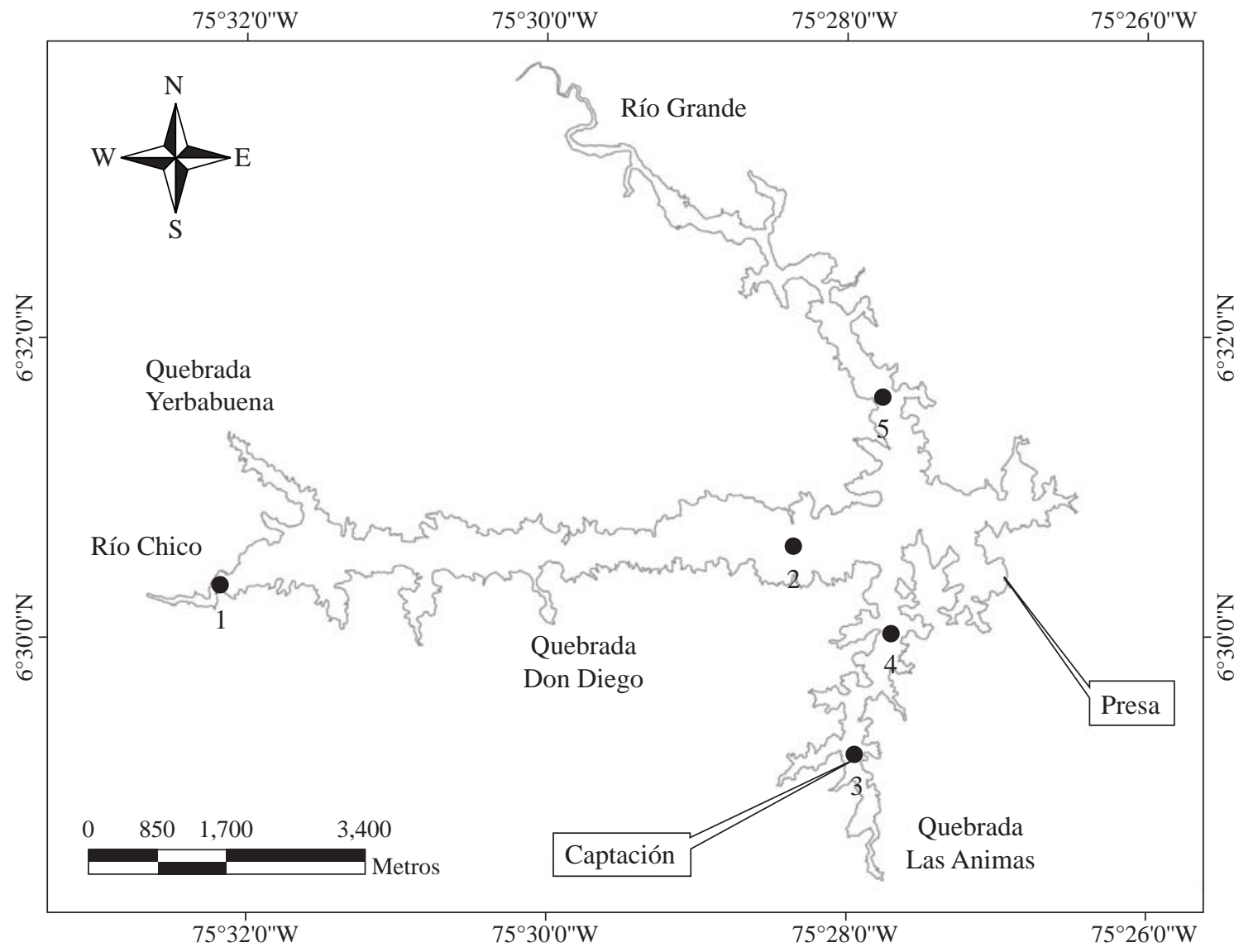

Figura 1. Ubicación del embalse Riogrande II y localización de las estaciones de muestreo

La información sobre la taxocenosis zooplanctónica se obtuvo de Villabona-González (2015).

\section{Tratamiento de la información}

Teniendo en cuenta que el tamaño celular del plancton es uno de los factores que controlan el metabolismo y la estructura de la comunidad (Chisholm, 1992; Rodríguez, 1994; Li, 2002), que la MDL es una medida indirecta de resistencia a la maniobrabilidad del zooplancton, y que se ha reportado una estrecha relación entre el contenido de carbono y el biovolumen para diferentes grupos del fitoplancton (Menden-Deuer \& Lessard, 2000; Cermeño, et al., 2005), para el cálculo de la concentración de carbono celular se clasificó el fitoplancton por rangos de tamaño según el promedio de la MDL como nanoplancton (5 a 20 $\mu \mathrm{m})$ y microplancton $(>20 \mu \mathrm{m})$.

El contenido de carbono celular (pgC/cel) se calculó mediante las ecuaciones de Menden-Deuer \& Lessard (2000), así: para algas no diatomeas, pgC/cel=0,216 x $\mathrm{V}^{0,939}$ y para diatomeas, $\mathrm{pgC} / \mathrm{cel}=0,288 \times \mathrm{V}^{0,811}$, donde $\mathrm{V}$ es el volumen celular. La biomasa total de carbono se obtuvo multiplicando el carbono celular por la densidad de células por mililitro (Gosselain \& Hamilton, 2000) y los resultados se reportaron en $\mathrm{pgC} / \mathrm{ml}$.
La fracción del fitoplancton que por su tamaño pudiera ser filtrado por el zooplancton reportado en el embalse Riogrande II (Villabona-González, 2015), se determinó según los criterios de Gophen \& Geller (1984), Kerner, et al. (2004) y Giraldo (2010), y coincidió en este caso con la porción nanoplanctónica, dado que según estos autores, la mayoría de los organismos filtradores que componen la taxocenosis pueden consumir partículas entre 0,5 y $35 \mu \mathrm{m}$, pero seleccionan especialmente las de tamaños inferiores a $20 \mu \mathrm{m}$.

Para estimar las concentraciones internas de nitrato (N) y fosfato (P) de las algas nanoplanctónicas y deducir la calidad de alimento que ofrecen al zooplancton de acuerdo con sus características bioquímicas, se calculó la concentración de nutrientes interna mínima en la que la tasa de crecimiento es igual a cero $\left(\mathrm{Q}_{\min }\right)$, así como la constante de saturación media para la absorción de nutrientes K (mmol nutrientes/l), utilizando las regresiones $\log _{10} \mathrm{Q}^{\mathrm{N}}$ min $=-8,7+0,68 * \log 10$ $\mathrm{V} ; \log _{10} \mathrm{~K}^{\mathrm{N}}=-0,71+0,52 * \log 10 \mathrm{~V}$ y $\log _{10} \mathrm{Q}_{\text {min }}^{\mathrm{P}}=-10,5$ $+0,86 * \log 10 \mathrm{~V}$, donde V es el volumen celular (Edwards, et al., 2012).

Debido a que la mayoría del zooplancton filtrador pertenecía al orden Cladocera, y que Bosmina freyi (Cladocera) representó el 60 \% de la biomasa zooplanctónica total 
(Villabona-González, 2015), para determinar el grado de asociación entre dicho orden y el nanoplancton, se ejecutó un análisis de correlación de Spearman utilizando el programa R, versión 3.2.0 (R Core Team, 2015).

\section{Resultados y discusión}

El microplancton $(>20 \mu \mathrm{m})$ representó $70,2 \%$ del fitoplancton, 99,1 \% del promedio del carbono celular por especie y $99,9 \%$ del promedio del carbono fitoplanctónico en el embalse (Tabla 1).

Según Chisholm (1992), ante condiciones saturadas de nutrientes, los fitopláncteres de células de MLD grande pueden generar florecimientos, mientras que los organismos pequeños tienden a permanecer relativamente constantes. Esto se debe a que en aguas ricas en nutrientes el fitoplancton de mayor tamaño tiene tasas más altas de producción de biomasa (Cermeño, et al., 2005; Marañón, et al., 2007), y es menos controlado por los herbívoros (Kiørboe, 1993).

En el microplancton la división Charophyta presentó los organismos con mayor volumen promedio y, por lo tanto, con mayor contenido de carbono celular. Dado que fue el grupo fitoplanctónico más abundante durante el estudio (López-Muñoz, 2015), su biomasa total de carbono superó ampliamente la de los demás y constituyó más de $98 \%$ del total en todos los muestreos y estaciones (Figura 2).

Debido a que el zooplancton filtrador del embalse está constituido principalmente por organismos pequeños en relación con el fitoplancton dominante $(0,2$ a $1,5 \mathrm{~mm}$ de longitud, según Villabona-González, 2015), y a que la morfología de los especímenes de Charophyta de mayor volumen y densidad (Staurastrum) le confiere ventajas para evitar el pastoreo (Hansson \& Tranvik, 1996), es posible considerar que la vía detrítica es el camino fundamental del flujo de energía en el embalse. Al respecto, Ramírez, et al. (2015) han señalado que en este sistema el tripton (organismos muertos, detritus y sustancias coloidales de origen orgánico o inorgánico) y no el fitoplancton es el componente que más contribuye con la atenuación de la irradiancia descendente, lo que sugiere una alta concentración de partículas detríticas y apoya esta presunción.

Según Rodríguez-Martínez (2010), en los ecosistemas acuáticos eutróficos las cadenas tróficas son cortas y la eficiencia de la transferencia de la producción primaria hacia los herbívoros es baja en comparación con las pérdidas hacia la vía detrítica. Sin embargo, la elevada producción primaria y el reducido número de niveles tróficos permiten la disponibilidad de biomasa para el resto de la cadena trófica. Se infiere, entonces, que en lagos eutróficos la principal fuente de alimento del zooplancton filtrador la constituyen el carbono orgánico detrítico y la biomasa bacteriana y, solo en muy baja proporción, el fitoplancton, y específicamente el nanoplancton (Geller \& Müller, 1981).

Pese a lo anterior y a que el énfasis de este trabajo se centró en estimar la biomasa fitoplanctónica disponible para los filtradores, es necesario considerar que los productores primarios planctónicos se consumen también como sustancias disueltas y no solo como alimento en partículas y que, en consecuencia, al considerar ambas vías se puede suponer que el flujo de energía indirecto desde el microplancton es alto, incluso teniendo en cuenta la exportación hacia el sedimento y las pérdidas por respiración bacteriana.

Además, en un estudio sobre la dieta del rotífero forrajero Asplanchna girodi en el embalse, Giraldo (2010) encontró que, aunque en menor proporción que las clorococales, las algas incluidas en la porción de microplancton, tales como Ceratium, Staurastrum, Coelastrum, y algunas diatomeas,

Tabla 1. Número de taxones por división, límites y valores medios de la máxima dimensión lineal (MDL), biovolumen, contenido de carbono por taxón y biomasa de carbono del fitoplancton del embalse Riogrande II por rangos de tamaño (agosto de 2011 a agosto de 2012)

\begin{tabular}{|c|c|c|c|c|c|c|}
\hline $\begin{array}{l}\text { Rango de } \\
\text { tamaño }\end{array}$ & División & $\begin{array}{l}\text { Número de } \\
\text { taxones }\end{array}$ & $\operatorname{MDL}(\mu \mathrm{m})$ & Biovolumen $\left(\mathrm{mm}^{3}\right)$ & Biomasa (pgC/cél) & Biomasa total $(\mathrm{pgC} / \mathrm{ml})$ \\
\hline \multirow{6}{*}{ Microplancton } & Charophyta & 15 & $23,9-455,9(90,8)$ & $0,1-832,2(208,8)$ & $8,8-78258,4(20314,8)$ & $2,1 * 10-1,8 * 10^{7}\left(1,8^{*} 10^{6}\right)$ \\
\hline & Chlorophyta & 19 & $20,3-149,2(47,8)$ & $0,0-16,7 \quad(5,2)$ & $3,8-1967,3$ & $2,4 * 10-1,5 * 10^{4}\left(2,1 * 10^{3}\right)$ \\
\hline & Cryptophyta & 1 & 21,7 & 2,8 & 369,2 & $2,7 * 10^{3}$ \\
\hline & Dinophyta & 3 & $31,1-193,7(92,6)$ & $17,5-69,0(50,0)$ & $2027,8-7534,2 \quad(5515,8)$ & $4,4 * 10^{3}-4,8^{*} 10^{4}\left(2,3 * 10^{4}\right)$ \\
\hline & Euglenophyta & 5 & $30,3-109,8(59,0)$ & $0,1-43,7(10,7)$ & $16,2-4903,6 \quad(1231,7)$ & $1,4 * 10-8,1 * 10^{3}\left(2,3 * 10^{3}\right)$ \\
\hline & Ochrophyta & 23 & $23,8-318,2(59,0)$ & $0,0-40,2(10,7)$ & $4,0-1196,4 \quad(1231,7)$ & $1,0 * 10-7,9 * 10^{3}\left(5,7 * 10^{2}\right)$ \\
\hline \multirow{5}{*}{ Nanoplancton } & Charophyta & 4 & $12,4-17,7(15,5)$ & $0,3-1,2 \quad(0,6)$ & $46,2-166,3$ & $8,5 * 10-2,8 * 10^{2}\left(1,7 * 10^{2}\right)$ \\
\hline & Chlorophyta & 14 & $8,7-17,7(12,8)$ & $0,0-3,0 \quad(0,6)$ & $2,2-396,8$ & $5,1-1,6 * 10^{3}\left(4,4 * 10^{2}\right)$ \\
\hline & Cryptophyta & 3 & $10,2-18,2(15,4)$ & $0,2-1,3 \quad(0,8)$ & $30,0-176,1$ & $3,7 * 10^{2}-7,3 * 10^{3}\left(3,3 * 10^{3}\right)$ \\
\hline & Euglenophyta & 2 & $15,4-18,3(16,8)$ & $2,2-3,5 \quad(2,9)$ & $295,0-455,3$ & $9,3 * 10^{2}-1,1 * 10^{3}\left(1,0 * 10^{3}\right)$ \\
\hline & Ochrophyta & 5 & $7,2-16,9(13,5)$ & $0,1-0,2 \quad(0,2)$ & $11,8-23,5$ & $2,6 * 10-1,8 * 10^{2}(6,7 * 10)$ \\
\hline
\end{tabular}


A.

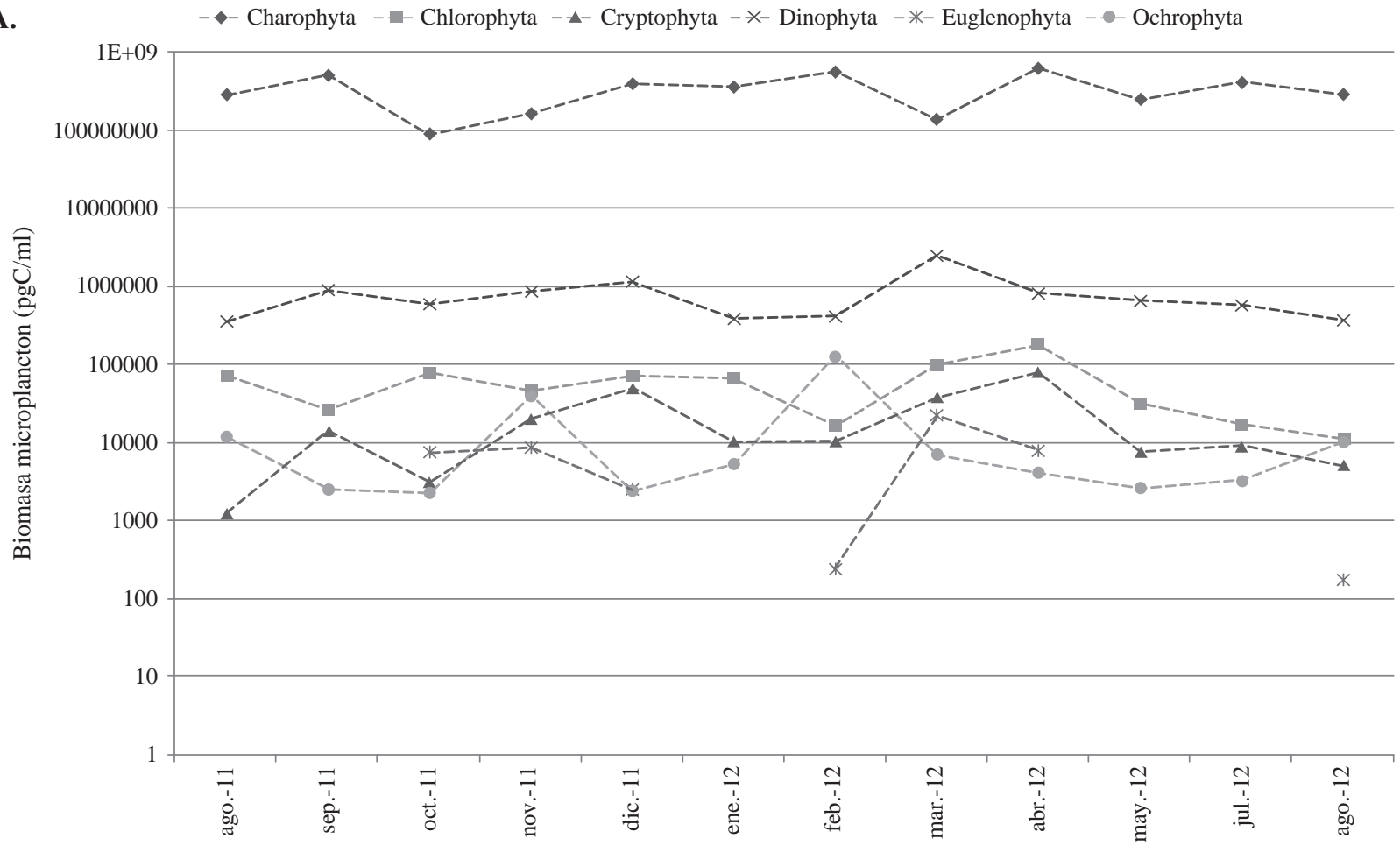

B.

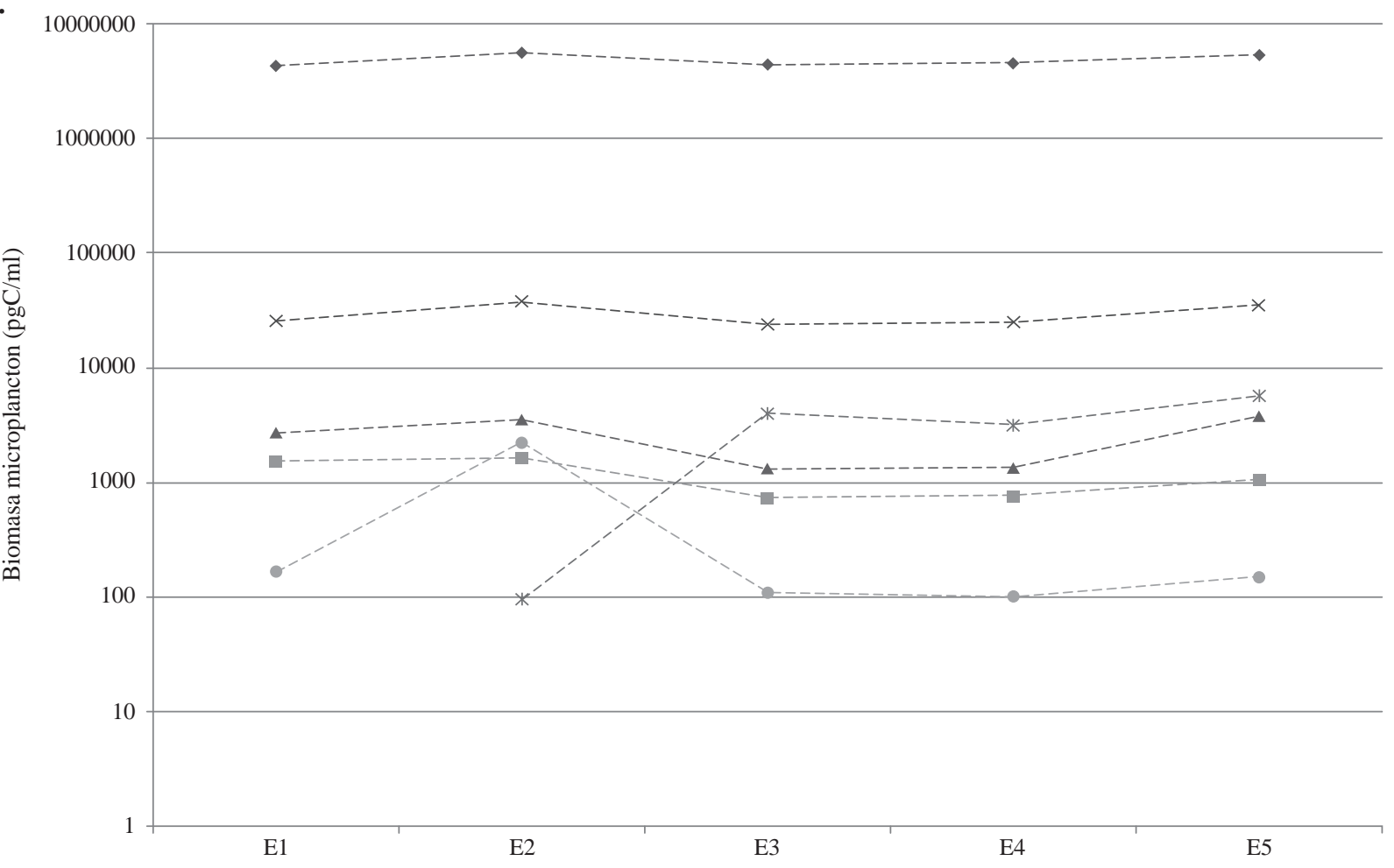

Figura 2. Biomasa de carbono del fitoplancton (microplancton) por divisiones taxonómicas. Embalse Riogrande II. A. Por muestreos. B. Por estaciones

hacen parte de la dieta de esta especie. De ellas, Ceratium y Staurastrum fueron dominantes durante este estudio y contribuyeron significativamente a la biomasa de carbono, como ya se mencionó.
El nanoplancton fue dominado por algas flageladas mixotróficas (Cryptophyta y, en menor proporción, Euglenophyta), cuya contribución a la biomasa de carbono en esta porción del fitoplancton superó el $70 \%$ en todas 
las muestras (Figura 3). En ambientes limitados de luz, la mixotrofia es una estrategia adaptativa que permite utilizar las bacterias como fuente de carbono orgánico y de nutrientes (Medina-Sánchez, 2002), lo que constituye una de las razones para que estas dos divisiones predominen en el nanoplancton.
Diversos autores han documentado la presencia de criptofitas en contenidos estomacales de especies del zooplancton. Además, Bogdan \& Gilbert (1987) encontraron una alta eficiencia en la ingestión de varias especies de Cryptophyta por parte de numerosos zooplancteres. De esta forma, es posible deducir que las especies nanoplanctónicas de

A.

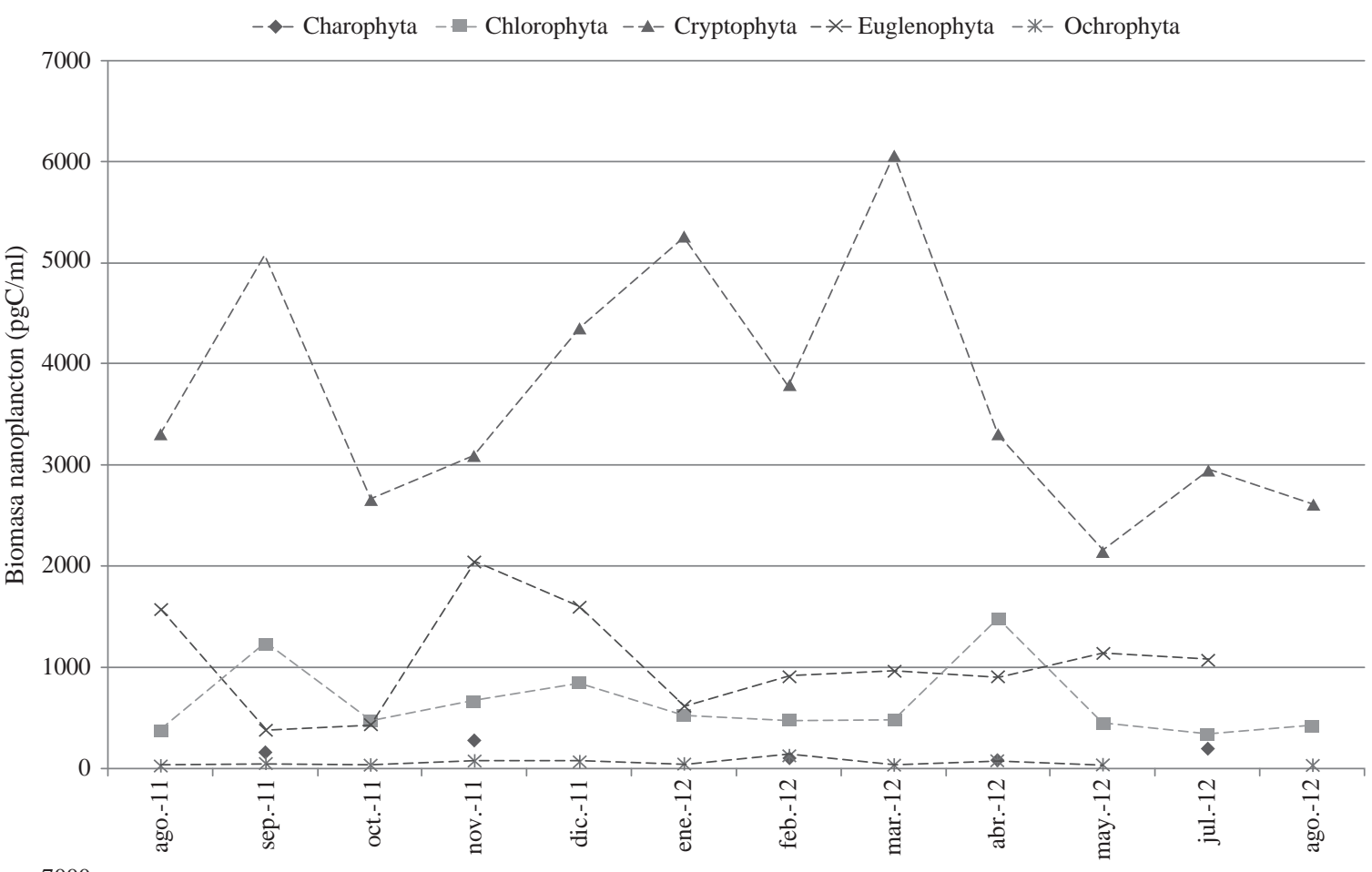

B.

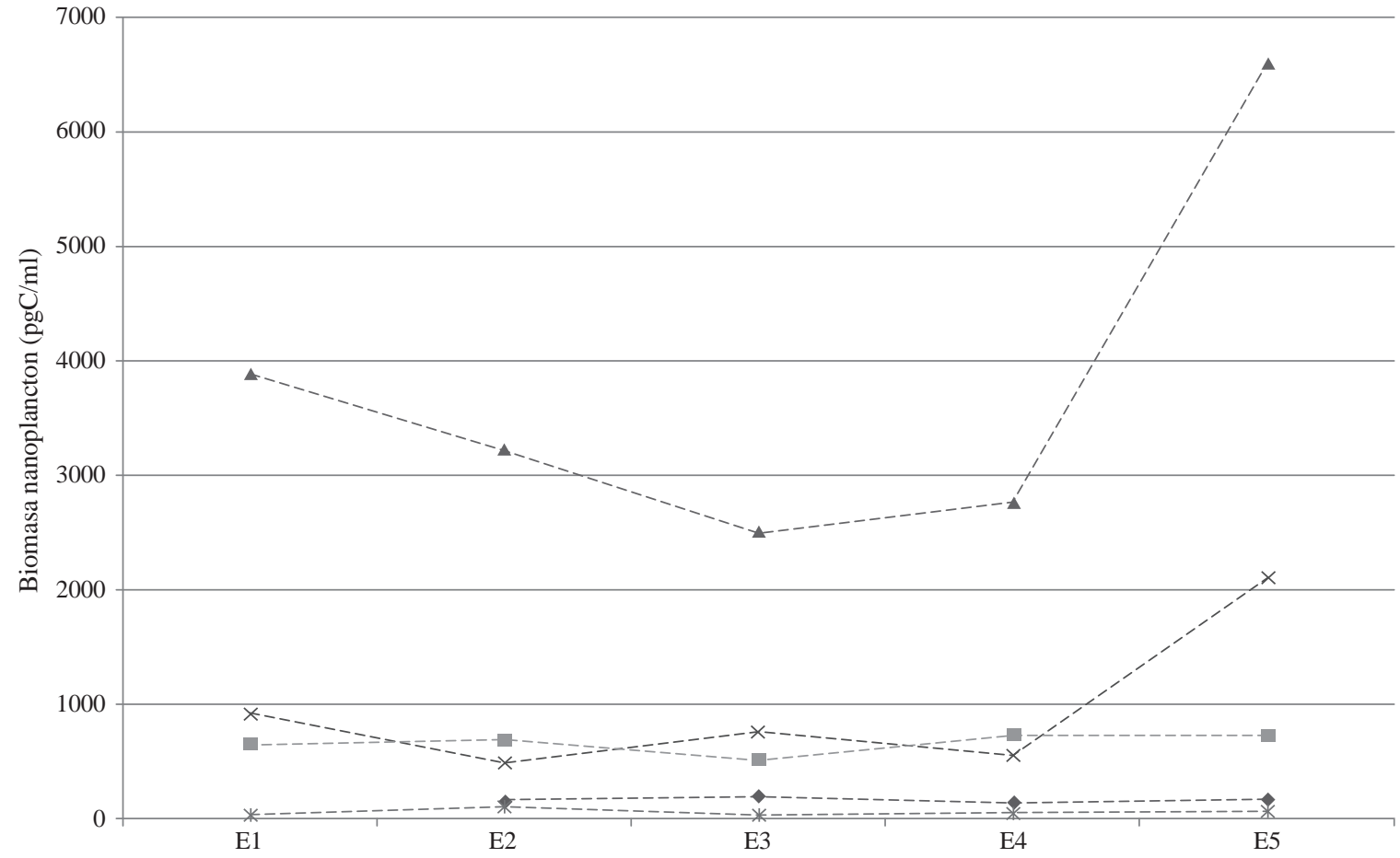

Figura 3. Biomasa de carbono del fitoplancton (nanoplancton) por divisiones taxonómicas. Embalse Riogrande II. A. Por muestreos. B. Por estaciones 
la división criptofita presentes en el embalse Riogrande II hacen parte de la dieta frecuente del zooplancton de este sistema.

En cuanto a la calidad de alimento que podría ofrecer el fitoplancton (nanoplancton), además de la biomasa de carbono, se evidenció que las divisiones Euglenophyta y Cryptophyta también pueden contener mayores concentraciones de nitratos y fosfatos (Tabla 2) y, por lo tanto, se asume que la ingestión de los organismos de estos grupos y la incorporación de tales nutrientes podría favorecer las tasas de crecimiento y la reproducción del zooplancton.

Aunque las biomasas del nanoplancton y los cladóceros no evidenciaron relaciones significativas (correlaciones entre 0,05 y 0,46 ), en la Figura 4 se muestra que la tendencia temporal de estas variables fue similar entre septiembre y noviembre de 2011 y entre marzo y abril de 2012. Además, espacialmente ambas taxocenosis presentaron las mayores biomasas en las estaciones localizadas al ingreso del brazo del río Chico (1) y al final del brazo del río Grande (5).

Debido a la poca biomasa de los organismos autótrofos en el nanoplancton, estos resultados pueden interpretarse como un efecto indirecto de la variación en la disponibilidad de recursos para las algas mixotróficas y el zooplancton filtrador (por ejemplo, bacterias, ciliados y protozoos nanoplanctónicos), tal como lo reportaron Hwang \& Heath (1997). Además, según Lampert \& Sommer (1997), la herbivoría del zooplancton es una de las principales fuentes de regeneración de nutrientes disueltos $\mathrm{y}$, por ende, uno de los factores que afecta las tasas de crecimiento del fitoplancton, pues una porción de los nutrientes contenidos en las algas es liberada a través de los detritus provenientes de la alimentación, las heces o la excreción animal.

Así mismo, en el plano espacial la tendencia hacia valores de biomasa más altos en las colas del embalse (ingreso del brazo del río Chico y final del brazo del río Grande), puede estar asociada a mayores concentraciones de material orgánico debido al ingreso de los afluentes y, en el caso

Tabla 2. Concentración interna mínima de los nutrientes (nitrato-N y fosfato-P) a la que la tasa de crecimiento algal es igual a cero $\left(\mathrm{Q}_{\min }\right)$, y constante de saturación media para la absorción de nutrientes (K) del fitoplancton (nanoplancton) del embalse Riogrande II (agosto de 2011 a agosto de 2012)

\begin{tabular}{lccc}
\hline División & $\mathbf{L o g}_{10} \mathbf{Q}_{\text {min }}^{\mathrm{N}}$ & $\mathbf{L o g}_{10} \mathbf{K}^{\mathrm{N}}{ }_{\text {min }}$ & $\mathbf{L o g}_{10} \mathbf{Q}_{\text {min }}^{\mathrm{P}}$ \\
\hline Charophyta & $-6,83$ & 0,72 & $-8,14$ \\
Chlorophyta & $-7,06$ & 0,55 & $-8,42$ \\
Cryptophyta & $-6,81$ & 0,73 & $-8,11$ \\
\hline Euglenophyta & $-6,36$ & 1,08 & $-7,54$ \\
\hline Ochrophyta & $-7,23$ & 0,41 & $-8,64$ \\
\hline
\end{tabular}

particular del ingreso del brazo del río Chico, por la mezcla provocada por la intrusión de la pluma del río hasta el fondo y por la poca profundidad de la columna de agua (Toro, et al., 2013), pues estos factores favorecen la disponibilidad de pequeñas partículas de alimento tanto para las algas mixótrofas (principales componentes del fitonanoplancton), como para el zooplancton.

Aunque la biomasa del fitonanoplancton fue notoriamente superior al final del brazo del río Grande, la de los cladóceros fue significativamente mayor en el ingreso del brazo del río Chico, lo cual puede ser el resultado de la alta sensibilidad de los flagelados mixotróficos a las floraciones de cianobacterias (Moustaka-Gouni, et al., 2006; Krev̌s, et al., 2010), ya que la mayor biomasa de cianobacterias se encontró en el ingreso del brazo del río Chico (PalacioBetancurt, 2014).

\section{Conclusiones}

En el embalse Riogrande II el fitoplancton eucariótico cumple un rol fundamental en la fijación del carbono, lo cual se refleja en los altos valores de biomasa, especialmente de organismos fototróficos. Sin embargo, debido a la eutrofia del sistema y a la pequeña talla del zooplancton dominante en comparación con la de las células algales, el flujo de energía, el de carbono y el de los nutrientes almacenados por las algas parece estar orientado principalmente a la vía detrítica y a las sustancias disueltas y no a los consumidores de niveles tróficos superiores, en este caso, el zooplancton filtrador.

En el fitoplancton analizado, la principal fuente de alimento para el zooplancton dominante parece estar constituida por organismos mixótrofos (Cryptophyta y Euglenophyta). No obstante, suponemos que la biomasa zooplanctónica está sustentada principalmente por algas y bacterias pertenecientes al nanoplancton (2 a $20 \mu \mathrm{m})$ y al picoplancton $(<2$ $\mu \mathrm{m})$, los cuales no fue posible detectar mediante el método de cuantificación empleado, y que, debido a su reducido biovolumen, contienen bajas concentraciones de carbono y nutrientes, aunque probablemente se encuentran en altas concentraciones en el embalse.

Lo anterior conduce a la aceptación parcial de la hipótesis planteada, pues aunque la porción de biomasa (estimada como carbono) disponible para el zooplancton fue inferior al $1 \%$ de la biomasa fitoplanctónica total y estuvo conformada principalmente por las especies agrupadas en Cryptophyta, más que las Chlorophyta, las Euglenophyta constituyeron el siguiente grupo de mayor contenido de carbono.

Los resultados obtenidos resaltan la necesidad de realizar estudios en los cuales se incluyan las fracciones de menor tamaño del fitoplancton y la taxocenosis de bacterioplancton, pues de esta forma se podrán realizar los cálculos necesarios para estimar el metabolismo y las transferencias de energía y carbono en los ecosistemas acuáticos. 

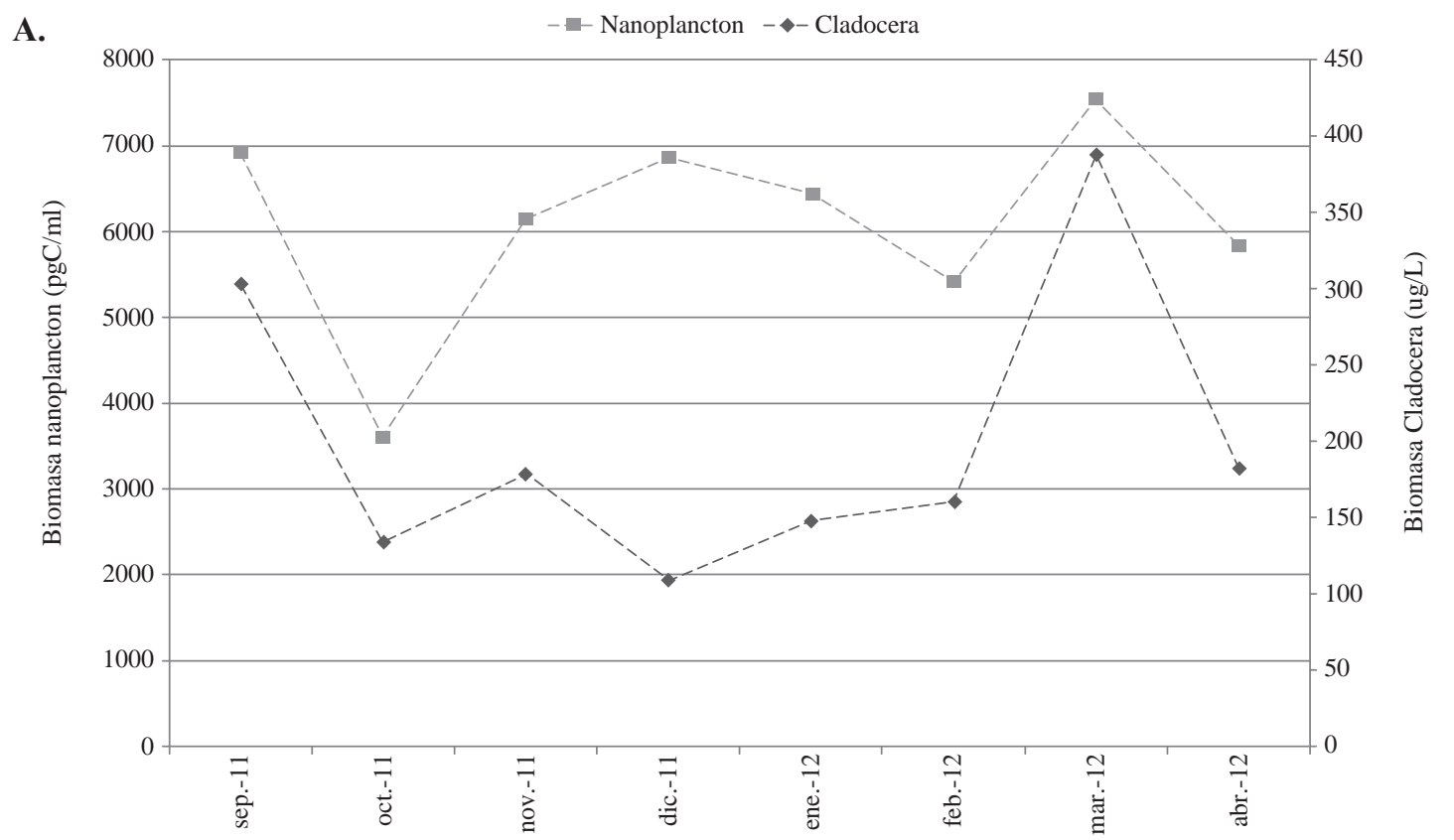

B.
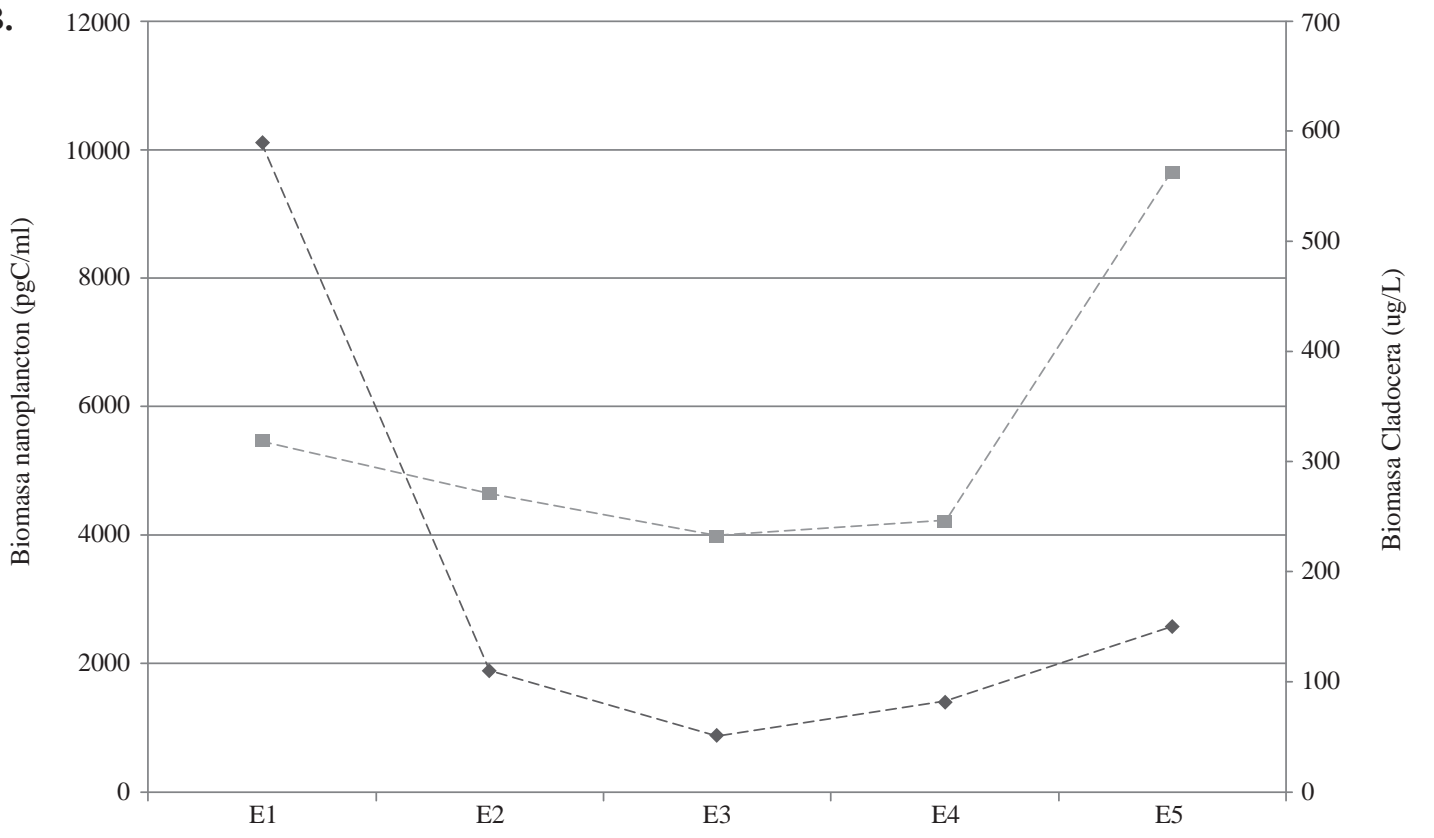

Figura 4. Variación de la biomasa media de carbono del fitoplancton (nanoplancton) y de la biomasa media de cladóceros en el embalse Riogrande II. A. Por muestreos. B. Por estaciones

\section{Agradecimientos}

Los autores agradecen a las Empresas Públicas de Medellín, por el financiamiento del programa de investigación "Estudio de las condiciones ambientales de tres embalses de Empresas Públicas de Medellín para la gestión integral y adecuada del recurso hídrico", del cual hace parte el presente estudio, y a Isabel Cristina Gil por la traducción al inglés.

\section{Conflicto de intereses}

Los autores declaran no tener conflicto de intereses.

\section{Bibliografía}

Bellinger E.G., Sigee D.C. 2010. Freshwater algae. Identification and use as bioindicators. Wiley-Black Well. John Wiley \& Sons. p. 271.

Bogdan, K.G., Gilbert, J.J. 1987. Quantitative comparison of food niches in some freshwater zooplankton. Oecología. 72: $331-340$

Bourrelly P. 1970. Les Algues d'Eau Douce. Initiation à la Systématique. Tome III, Les algues blues et rouges: Les Eugléniens, Peridiniens et Cryptomonadines. Éditions N. Boubée. Paris. p. 572. 
Castro, A.J., Bicudo, C.E. de M. 2007. Flora ficológica. Cryptophyceae. Rima. p. 121.

Cermeño, P., Estévez-Blanco, P., Marañón, E., Fernández, E. 2005. Maximum photosynthetic efficiency of sizefractionated phytoplankton assessed by ${ }^{14} \mathrm{C}$-uptake and fast repetition rate fluorometry. Limnol. Oceanogr. 50 (5): 1438-1446.

Chisholm S.W. 1992. Phytoplankton size. En: Falkowski, P.G \& Woodhead, A.D. (Eds.). Primary productivity and biogeochemical cycles in the sea. Plenum, New York. p. 213-237.

Comas A. 1996. Las Chlorococcales Duliacuícolas de Cuba. Bibliotheca Phycologia, 99. Cramer, Stuttgart. p. 192.

Cox E.J. 1996. Identification of Freshwater Diatoms from live Material. Chapman \& Hall, London. p. 168.

Edwards, K.F., Thomas, M.K., Klausmeier, C.A., Litchman, E. 2012. Allometric scaling and taxonomic variation in nutrient utilization traits and maximum growth rate of phytoplankton. Limnol. Oceanogr. 57 (2): 554-566.

Gasse F. 1986. East African diatoms. Taxonomy, ecological distribution. Bibliotheca Diatomologica. J. Crammer. Berlin - Stuttgart. p. 201.

Geller, W., Müller, H. 1981. The filtration apparatus of Cladocera: Filter mesh-sizes and their implications on food selectivity. Oecología. 49: 316-321.

Giraldo, M. 2010. Composición de la dieta del rotífero Asplanchna girodi (De Guerne, 1888) y su variación temporal y espacial en el embalse Riogrande II (Antioquia, Colombia). Trabajo de pregrado, Instituto de Biología, Universidad de Antioquia. Medellín, Colombia.

Gophen, M., Geller, W. 1984. Filter mesh size and food particle uptake by Daphnia. Oecologia. 64: 408-412.

Gosselain, V., Hamilton, P. B. 2000. Algamica: Revisions to a key-based computerized counting program for free-living, attached, and benthic algae. Hydrobiologia. 438: 139-142.

Hansson, L.A., Tranvik, L.J. 1996. Quantification of invertebrate predation and herbivory in food chains of low complexity. Oecologia. 108: 542-551.

Hillebrand, H., Duerselen, C.D., Kirschtel, D., Pollingher, U., Zohary, T. 1999. Biovolume calculation for pelagic and benthic microalgae. J. Phycol. 35: 403-424.

Huber-Pestalozzi, G. 1968. Das Phytoplankton des Süsswassers. Systematik und Biologie. 3. Teil: Cryptophyceae, Chloromonadophyceae, Dinophyceae. E. Schweizerbart'sche Verlagsbuchhandlung (Nägele u. Obermiller), Stuttgart, Germany. p. ¿ं?

Hwang, S.J., Heath, R. 1997. The distribution of protozoa across a trophic gradient, factors controlling their abundance and importance in the plankton food web. J. Plankton Res. 19: 491-518

Kerner, M., Ertl, S., Spitzy, A. 2004. Trophic diversity within the planktonic food web of the Elbe Estuary determined on isolated individual species by ${ }^{13} \mathrm{C}$ analysis. J. Plankton Res. 26 (9): 1039-1048.
Kiørboe, T. 1993. Turbulence, phytoplankton cell size, and the structure of pelagic food webs. Adv. mar. Biol. 29: 1-72.

Komárek J., Fott B. 1983. Das Phytoplankton des SüBwassers. E. Schweizerbart'sche Verlagsbuchhandlung. Stuttgart. p. 1044.

Krevš, A., Koreivienė, J., Mažeikaitė, S. 2010. Plankton food web structure during cyanobacteria bloom in the highly eutrophic Lake Gineitiškès. Ekologija. 56 (1/2): 47-54.

Lampert, W., Sommer, U. 1997. Limnoecology: The ecology of lakes and streams. Oxford University Press. New York. p. 382 .

Li-W, K.W. 2002. Macroecological patterns of phytoplankton in the northwestern North Atlantic Ocean. Nature. 419: 154-157.

López-Muñoz, M.T. 2015. Aspectos taxonómicos y ecológicos del fitoplancton eucariótico del embalse Riogrande II (Antioquia, Colombia). Tesis de doctorado, Facultad de Ciencias Exactas y Naturales. Instituto de Biología. Programa de Posgrado. Universidad de Antioquia. Medellín, Colombia.

Lund, G., Kippling, C., Le Cren, E.D. 1958. The inverted microscope method of estimating algal numbers and analysis statistical basis of estimation by counting. Hidrobiologia. 11: 143-170.

Marañón, E., Cermeño, P., Rodríguez, J., Zubkov, M.V., Harris, R.P. 2007. Scaling of phytoplankton photosynthesis and cell size in the ocean. Limnol. Oceanogr. 52 (5): 2190-2198.

Medina-Sánchez J.M. 2002. El eslabón fitoplancton - bacteria en un ecosistema de alta montaña: estructura, función y regulación. Tesis doctoral, Departamento de Biología animal y ecología. Universidad de Granada, España.

Menden-Deuer, S., Lessard, E.J. 2000. Carbon to volume relationships for dinoflagellates, diatoms, and other protist plankton. Limnol. Oceanogr. 45 (3): 569-579.

Moustaka-Gouni, M., Vardaka, E., Michaloudi, E., Kormas, K.A., Tryfon, E., Mihalatou, H., Gkelis, S., Lanaras, T. 2006. Plankton food web structure in a eutrophic polymictic lake with a history in toxic cyanobacterial blooms. Limnol. Oceanogr. 51 (1/2): 715-727.

Moss, B., Mckee, D., Atkinson, D., Collings, S.E., Eaton, J.W., Gill, A.B., Harvey, I., Hatton, K., Heyes, T., Wilson, D. 2003. How important is climate? Effects of warming, nutrient addition and fish on phytoplankton in shallow lake microcosms. Journal of Applied Ecology. 40: 782-792.

Palacio-Betancurt H.M. 2014. Dinámica espacio-temporal de las cianobacterias en el embalse Riogrande II. Tesis de doctorado, Facultad de Ciencias Exactas y Naturales. Instituto de Biología. Programa de Posgrado. Universidad de Antioquia. Medellín, Colombia.

Prescott G.W., Croasdale H.T., Vinyard W.C. 1972. North American Flora. The New York Botanical Garden. p. 84.

Prescott G.W., Croasdale H.T., Vinyard W.C. 1975. A Synopsis of North American Desmids. Part II. Desmidiaceae: Placodermae. Section 1. University of Nebraska Press. USA. p. 275. 
Prescott G.W., Croasdale H.T., Vinyard W.C., Bicudo C.E. de M. 1982. A Synopsis of North American Desmids. Part II. Desmidiaceae: Placodermae. Section 4. University of Nebraska Press. USA. p. 700.

R Core Team. 2015. R: A language and environment for statistical computing. R Foundation for Statistical Computing, Vienna, Austria. URL. http://www.R-project.org.

Ramírez, J.J., Arcila, M.C., Sepúlveda,S.C. 2015. Cuantificación de los componentes que afectan el coeficiente de atenuación vertical para irradiancia descendente en el embalse Riogrande II (Colombia). Rev. Acad. Colomb. Cienc. Ex. Fis. Nat. 39 (151): 228-238.

Rocha, O., Duncan, A. 1985. The relationship between cell carbon and cell volume in freshwater algal species used in zooplanktonic studies. J. Plankton Res. 7 (2): 279-294.

Rodríguez, J. 1994. Some comments on the size-based structural analysis of the pelagic ecosystem. Sci. Mar. 58 (1-2): 1-10.

Rodríguez-Martínez J. 2010. Ecología. 2 Edición. Ediciones Pirámide. Madrid, España.

Ros J. 1979. Prácticas de Ecología. Ed. Blume. Barcelona, España.

Sigee D.C. 2004. Freshwater Microbiology: Diversity and dynamic interactions of microorganisms in the aquatic environment. Chichester, UK, John Wiley \& Sons. p. 544.

Teiling E. 1967. The desmid genus Staurodesmus. A taxonomic study. Arkiv För. p. 629.

Tell G., Conforti V. 1986. Euglenophyta pigmentadas de la Argentina. Bibliotheca Phycologica, 75: Berlin-Stuttgart, Ed. Cramer. p. 301.
Toro, M., Moreno, A., Chalarca, D., Grajales, H. 2013. Dinámica ambiental de los nutrientes (especies químicas del nitrógeno, fósforo y sílice) en los embalses $\mathrm{La} \mathrm{Fe}$, Riogrande II y Porce II a través de modelos conceptuales y matemáticos, como herramienta de gestión de la calidad de agua en los tres sistemas. En: Palacio J (ed), Informe técnico embalse Riogrande II "Estudio de la problemática ambiental de tres embalses de Empresas Públicas de Medellín para la gestión integral y adecuada del recurso hídrico". Universidad de Antioquia, Universidad Nacional sede Medellín, Empresas. Públicas de Medellín. Medellín: 242-297.

Utermöhl, H. 1958. Zur Vervollkommnung der quantitativen Phytoplankton-methodik. Mitt int. Ver. Limnol. 9: 1-38.

Villabona-González S.L. 2015. Relaciones ecológicas de la biomasa de las poblaciones de rotíferos y microcrustáceos en la zona fótica del embalse Riogrande II, Antioquia, Colombia. Tesis de doctorado, Facultad de Ciencias Exactas y Naturales. Instituto de Biología. Programa de Posgrado. Universidad de Antioquia. Medellín, Colombia.

Vollenweider R.A. 1969. A manual on methods for measuring primary production in aquatic environments. I.B.P. Handbook 12. Davis F.A. Co. Blackwell Sci. Pub. Oxford. p.213.

West W., West G.S., Carter N. 1923. A Monograph of the British Desmidiaceae. Vol. 5. The Ray Society. London. p. 299. 